\title{
High Concentration of Spermine Induces the Dedifferentiation of Somatic Cells into Pluripotent Stem Cells
}

\section{Naofumi Shiomi}

School of Human Sciences, Kobe College, Nishinomiya, Japan

Correspondence to: Naofumi Shiomi, n-shiomi@mail.kobe-c.ac.jp

Keywords: Polyamine, Spermine, Pluripotent Stem Cells, Dedifferentiation, Cancer Stem Cells, Regenerative Medicine Received: January 20, $2019 \quad$ Accepted: February 18, $2019 \quad$ Published: February 21, 2019

Copyright () 2019 by authors and Scientific Research Publishing Inc.

This work is licensed under the Creative Commons Attribution International License (CC BY 4.0).

http://creativecommons.org/licenses/by/4.0/

\section{(c) (i) Open Access}

\section{ABSTRACT}

Cancer tissues contain cancer stem cells (CSCs), which play important roles in cancer metastasis. However, the mechanisms through which cancer cells dedifferentiate into stem cells have not yet been elucidated. In this study, the effects of high concentrations of polyamines produced in cancer cells on dedifferentiation were examined. The results showed that when normal human fibroblasts were cultured with high concentrations of spermine, the obtained polyamine-induced cells expressed alkaline phosphatase and marker proteins of pluripotent stem cells, although apoptosis occurred in most cells. In contrast, another polyamine-induced stem (PIS) cell line (Spe-2 PIS cells), obtained by culture in medium containing Rock, p53, and Bax inhibitors plus spermine, did not show signs of apoptosis. These Spe-2 PIS cells expressed marker proteins of pluripotent stem cells and differentiated into cardiomyocytes, brown adipocytes, and nerve cells. These results suggest that a high concentration of spermine, which often induces apoptosis in normal cells, has the capacity to dedifferentiate somatic cells into pluripotent stem cells and may be associated with the dedifferentiation of cancer cells, which continuously produce high concentrations of spermine. Moreover, the procedure to obtain Spe-2 PIS cells, which is simple and efficient, may have potential applications in regenerative medicine.

\section{INTRODUCTION}

Polyamines are aliphatic amines containing more than two amino groups; putrescine, spermidine, and spermine are the primary polyamines in mammals [1-3]. Polyamines bind to chromosomal DNA, mRNA, and proteins and play important roles in translation, gene expression, protein phosphorylation, and signal transduction $[4,5]$. Moreover, polyamines have positive effects on proliferation when added to 
cell cultures [3,6] and protect cells from the harmful effects of reactive oxygen species [7].

Because of their important physiological roles, the concentrations of polyamines are strictly controlled in cells via various metabolic pathways [8]. For example, putrescine is synthesized from ornithine by ornithine decarboxylase (ODC). Spermidine and spermine are synthesized from both putrescine and decarboxylated S-adenosylmethionine via adenosylmethionine decarboxylase (AdoMetDC), spermidine synthetase, and spermine synthetase. Spermidine and spermine are degraded to putrescine via $\mathrm{N}$-acetylspermine and $\mathrm{N}$-acetylspermidine by spermine/spermidine $\mathrm{N} 1$-acetyl transferase (SAT) and polyamine oxidase. The activities of AdoMetDC, ODC, and SAT are strictly controlled to maintain polyamines at low levels under normal conditions and are enhanced only when polyamines at higher levels are necessary. However, these mechanisms are disrupted in cancer cells, which continuously produce high amounts of polyamines, such as spermidine and spermine $[9,10]$. Cancer cells then exploit the functions of polyamines; for example, high concentrations of polyamines in cancer cells enhance proliferation and inhibit the expression of $\mathrm{CD} 44$, a molecule that binds to the extracellular matrix, thereby enhancing migratory ability [11]. Consistent with this, patients with cancer exhibit high levels of acetylpolyamines in the blood, and the levels of these compounds can be normalized after excision of the tumor.

Cancer stem cells (CSCs) contained in cancer tissues were recently found to be a major cause of recurrence in cancer therapy [12-14], and researchers are currently evaluating the characteristics of CSCs. However, the mechanisms through which cancer cells produce CSCs by dedifferentiation have not yet been elucidated. I hypothesized that dedifferentiation may be induced by polyamines, since cancer cells are exposed to high concentrations of spermidine and spermine in cancer tissues. Thus, in this study, the effects of high concentrations of spermine on cell dedifferentiation were examined. Normal somatic cells were used for experiments in place of cancer cells because cancer cells secrete various components that also affect the characteristics of cancer cells. The results provide important insights into the capacity of polyamine-induced stem (PIS) cells to differentiate into various types of cells.

\section{MATERIALS AND METHODS}

\subsection{Chemicals}

Putrescine, spermidine, and spermine (product codes: 51799-100MG, S2626-25G, and S3256-25G, respectively; Sigma-Aldrich Japan, Tokyo, Japan) were used as polyamines. These compounds were dissolved in distilled water at final concentrations of 20 and $100 \mathrm{mM}$, and the solutions were then sterilized with a $0.2-\mu \mathrm{m}$ membrane filter (Steradisc13; product code: S-1302; Kurabo Industries Ltd., Osaka, Japan). Y-27632 (CAS: 129830-38-2; product code: 039-24591; Wako Pure Chemical Industries, Ltd., Osaka, Japan) and thiazovivin (CAS: 5-11296-88-1; product code: 202-18011; Wako Pure Chemical Industries, Ltd.) were used as Rho-associated kinase (Rock) inhibitors [15, 16]. Bax-inhibiting peptide V [17] (CAS: 579492-81-2; product code: B1436-5MG; Sigma-Aldrich Japan) and the cyclic pifithrin- $\alpha$-hydrobromide [18] (CAS: 511296-88-1; product code: C2826; Tokyo Chemical Industry Co., Ltd., Tokyo, Japan) were used as Bax and p53 inhibitors of apoptosis. Y-27632 and Bax inhibitor peptide V were dissolved in distilled water at final concentrations of 30 and $3.4 \mathrm{mM}$, and thiazovivin and cyclic pifithrin- $\alpha$-hydrobromide were dissolved in dimethyl sulfoxide (CultureSure DMSO; grade: cell culture; product code: 031-24501, Wako Pure Chemical Industries, Ltd.) and 99.5\% ethanol (CultureSure Ethanol; grade: cell culture; product code: 039-24971; Wako Pure Chemical Industries, Ltd.) at final concentrations of 10 and $4.0 \mathrm{mM}$. These inhibitors were sterilized with $0.2-\mu \mathrm{m}$ membrane filters. TrypLE Select (product code: 12563029; Thermo Fisher Scientific, Tokyo, Japan) and STEM-CELLBANKER (GMP grade; product code: CB047; Takara Bio Inc., Shiga, Japan) were used for collection and storage of PIS cells, respectively. Hoechst 33342 solution (product code: 346-07951; Dojindo Laboratories, Kumamoto, Japan) and Actin Red 555 Ready Probes Reagent (product code: R37112; Thermo Fisher Scientific) were used for the staining of chromosomes and F-actin, respectively.

\subsection{Antibodies}

Anti-human OCT4A and SOX2 rabbit monoclonal antibodies; anti-human SSEA-4, TRA-1-60, and 
TRA-81 mouse monoclonal antibodies; and anti-mouse SSEA-1 mouse monoclonal antibody (product codes: ASA-0110, 0120, 0150, 0160, 0170, and 0135, respectively; Applied Stem Cell Inc., Milpitas, CA, USA) provided by Human and Mouse ES/iPS Cell Characterization Kits were used for the detection of pluripotent stem cells. Anti-human OTX2, anti-brachyury, and anti-SOX17 goat polyclonal antibodies (product codes: part\#967331, 967332, and 967330, respectively; R \& D Systems, Inc., Minneapolis, MN, USA) provided by StemXVivo kits were used for the detection of ectoderm, mesoderm, and endoderm, respectively. Anti-human cardiac troponin $\mathrm{T}(\mathrm{cTnT})$ mouse monoclonal (troponin $\mathrm{T}$, cardiac isoform Ab-1 [clone 13-11]; product code: \#MS-295-P0; Thermo Fisher Scientific); anti-sarcomeric alpha actinin rabbit polyclonal (product code: bs-10367R; Funakoshi Co. Ltd., Tokyo, Japan); anti-human and mouse uncoupling protein 1 (UCP1) rabbit polyclonal (product code: bs-1925R; Funakoshi Co. Ltd.); anti- $\beta$-tubulin III rabbit polyclonal, anti-myelin basic protein (MBP) rabbit polyclonal, and anti-microtubule-associated protein (MAP2) rabbit polyclonal antibodies (product codes: T3952, M3821, and M3696, respectively; Sigma-Aldrich Japan) were used for the detection of cardiomyocytes, brown adipocytes, and nerve cells. Anti-rabbit IgG goat polyclonal antibodies conjugated with $\mathrm{Cy}-3$ (product code: AP132C; Merck, Osaka, Japan), anti-mouse IgG rabbit polyclonal antibodies conjugated with Alexa Fluor 555 (product code: ab150126; Abcam Japan, Tokyo, Japan), and anti-goat IgG donkey polyclonal antibodies conjugated with Alexa Fluor 555 (product code: ab150130; Abcam Japan) were used as secondary antibodies. Use of these secondary antibodies enables the visualization of proteins bound to primary antibodies, which appear red under a fluorescent microscope (EVOS FL Auto Cell Imaging System; Thermo Fisher Scientific).

\subsection{Cells and Medium}

TIG-1-20 fibroblasts acquired from the JCRB Cell Bank (cell number: JCRB0501, lot number: 0472015) [19] were used as a typical somatic cell line. TIG-1-20 fibroblasts represent normal human lung fibroblasts obtained from a 5-year-old girl and established by the Tokyo Metropolitan Institute of Gerontology and they have been widely used for aging studies. These cells had a population doubling level (PDL) of 23.5 when first obtained; the PDL of the cells used in this study was around 30-35. HepG2 cells were acquired from the JCRB Cell Bank (cell number: JCRB1054, lot number: 05272013). HepG2 cells constitute a human hepatocellular carcinoma cell line derived from a 15-year-old American male and established by B. B. Knowless that has been widely used for cancer studies. HepG2 cells were used as representative cancer cells in this study. The cell lines of TIG-1-20 and HepG2 did not correspond to cross-contaminated strains in the data base of International Cell Line Authentication Committee (ICLAC). To avoid cross-contamination between TIG-1-20 and HepG2 cells, the cell lines were cultured separately in two different $\mathrm{CO}_{2}$ incubators, and the shape of TIG-1-20 cells on the culture plates was checked under a microscope before dedifferentiation experiments to detect potential contamination by HepG2 cells. 3T3-L24 murine white adipocytes constitute a cell line obtained by differentiation from 3T3-L1 murine pre-adipocytes in my laboratory. The characteristics of these cells as mouse adipocytes were examined in detail in a previous study [20], and these cells were used as representative mouse cells. The 3T3-L1 murine pre-adipocytes were purchased from the ECACC Cell Bank (cell number: EC86052701) in 2005; this cell line was established by H. Green et al. and has been widely used in adipose studies. ReproCardio 2 cardiomyocytes, purchased from ReproCell Co. Ltd. (code number: RCDC001N, Yokohama, Japan) and produced by differentiation from induced pluripotent stem (iPS) cells, were used as a positive control for cardiomyocytes.

Four basic media were used for culture. Modified Eagle's medium with Earle's salts and L-glutamine without sodium bicarbonate (product code: 1010120; MP Biochemical Inc., Illkirch, France) containing sodium bicarbonate (product code: S8761-100ML; Sigma-Aldrich Japan) was mixed with fetal bovine serum (FBS) at a ratio of 9:1 (10\% FBS). The mixture (MEM) was used for the culture of TIG-1-20 fibroblasts and HepG2 cancer cells. Cellartis DEF-CSTM 500 Culture Medium (product code: Y30010; Takara Bio Inc.) was used to grow human PIS cells. Plates or dishes were pre-coated with Coat-1, provided by the 
kit, before culture. Dulbecco's modified Eagle's medium (product code: 1033120; MP Biochemical Inc.) containing sodium bicarbonate was mixed with FBS at a ratio of 9:1. The mixture (DMEM) was then used for the culture of 3T3-L24 adipocytes. Stem Medium, a serum-free medium for mouse embryonic stem (ES) cells (product code: DSRK100; DS Pharma Biomedical, Osaka, Japan) containing leukemia inhibitory factor (StemSure LIF; grade: cell culture; product code: 199-16051; Wako Pure Chemical Industries, Ltd.), was use for the culture of mouse PIS cells. Penicillin-streptomycin solution (grade: cell culture; product code: 168-23191; Wako Pure Chemical Industries, Ltd.) was added to all media at a final concentration of 100 units $/ \mathrm{mL}$ penicillin and $100 \mu \mathrm{g} / \mathrm{mL}$ streptomycin. Cells were cultured in $\mathrm{CO}_{2}$ incubators $\left(5.0 \% \mathrm{CO}_{2}\right)$ at $37^{\circ} \mathrm{C}$.

\subsection{Effects of High Spermine Concentrations on Fibroblasts}

TIG-1-20 fibroblasts (5000 cells) were cultured in 24-well plates containing $2 \mathrm{~mL} /$ well MEM for $12 \mathrm{~h}$. Spermine solution was then added at a final concentration of $40 \mu \mathrm{M}$ (value determined from preliminary experiments), and cells were cultured for 1 day. Morphological changes in TIG-1-20 fibroblasts cultured for 0,12 , and $24 \mathrm{~h}$ were observed under a microscope. Alkaline phosphatase (AP) activity in TIG-1-20 fibroblasts cultured in the same medium for 0,12 , and $24 \mathrm{~h}$ was determined using an AP Staining Kit (product code: \# AP100R-1; System Biosciences Inc., Palo Alto, CA, USA) according to the manufacturer's instructions. Cells expressing AP were stained red.

\subsection{PIS Cells}

Spe-1 PIS cells were obtained as follows. Briefly, TIG-1-20 fibroblasts $\left(1 \times 10^{5}\right.$ cells $)$ were cultured in $25-\mathrm{cm}^{2}$ culture flasks containing $6 \mathrm{~mL}$ MEM $+40 \mu \mathrm{M}$ spermine for $24 \mathrm{~h}$. Many cells were suspended during culture upon losing their adherent phenotype, and both suspended and weakly attached cells were harvested by centrifugation $(800 \times \mathrm{g}, 3 \mathrm{~min})$. Cells were cultured in 6 -well plates containing $4 \mathrm{~mL}$ Cellartis DEF-CSTM 500 Culture medium for 2 - 3 days. Cells that were attached or weakly attached to the plates were removed by pipetting or with TrypLE select and harvested by centrifugation $(800 \times \mathrm{g}, 3 \mathrm{~min})$. The obtained strain was named "Spe-1".

Spe-2, PIS-Hep2, and PIS-L24 cells were obtained as follows. Briefly, MEM (or DMEM) $+20 \mu \mathrm{M}$ $\mathrm{Y} 27632+5 \mu \mathrm{M}$ thiazovivin $+20 \mu \mathrm{M}$ cyclic pifithrin- $\alpha$ hydrobromide $+30 \mu \mathrm{M}$ BAX-inhibiting peptide V5 (Rock/apoptosis-inhibiting medium) was used as a basic medium for induction. TIG-1-20 fibroblasts $(1 \times$ $10^{5}$ cells) were cultured in $25-\mathrm{cm}^{2}$ culture flasks containing $6 \mathrm{~mL}$ Rock/apoptosis-inhibiting medium for 16 $\mathrm{h}$ and were subsequently cultured for 1 day after adding $20 \mu \mathrm{M}$ spermine. During culture, $40-60 \mu \mathrm{M}$ spermine was added every day (final concentration of $100-160 \mu \mathrm{M}$ spermine). HepG2 hepatocellular carcinoma cells and 3T3-L24 white adipocytes $\left(1 \times 10^{5}\right.$ cells $)$ were cultured in $25-\mathrm{cm}^{2}$ culture flasks containing $6 \mathrm{~mL}$ Rock/apoptosis-inhibiting medium for $16 \mathrm{~h}$ and were subsequently cultured with the addition of $50 \mu \mathrm{M}$ spermine every day (final concentration of $100 \mu \mathrm{M}$ spermine). The obtained PIS cells were removed with TrypLE Select and harvested by centrifugation $(800 \times \mathrm{g}, 3 \mathrm{~min})$, and the resulting cells were cultured in 6-well plates containing $4 \mathrm{~mL} /$ well Cellartis DEF-CSTM 500 Culture medium for 2 - 3 days. The three obtained PIS cell lines induced from TIG-1-20, HepG2, and 3T3-L24 cells were named Spe-2, PIS-Hep2, and PIS-L24, respectively. These cell lines were directly used for subsequent experiments or stored with STEM-CELLBANKER at $-80^{\circ} \mathrm{C}$ until use.

\subsection{Apoptosis of Spe-1 PIS Cells and Effects of Rock Inhibitors}

TIG-1-20 fibroblasts were cultured in 24-well plates containing $2 \mathrm{~mL} /$ well $\mathrm{MEM}+40 \mu \mathrm{M}$ spermine for 12 or $36 \mathrm{~h}$. After culture for $12 \mathrm{~h}$, cells were used for analysis of membrane dysfunction with an APOPercentage Apoptosis Assay kit (product code: A0500; Biocolor Ltd., Newtownabbey, Northern Ireland) according to the manufacturer's instructions. APOPercentage Dye, a red dye provided in the Apoptosis Assay kit, can pass through cell membranes only in apoptotic cells that have lost flippase activity, staining apoptotic cells pink. In cells cultured for $36 \mathrm{~h}$, staining was carried out for detection of chromosomes and 
F-actin using Hoechst 33342 solution and Actin Red 555 Ready Probes Reagent, respectively. The stained cells were observed with a fluorescence microscope (EVOS FL Auto Cell Imaging System). Rock inhibitor-treated cells were obtained by culturing TIG-1-20 cells $\left(1 \times 10^{5}\right.$ cells $)$ in $25-\mathrm{cm}^{2}$ culture flasks containing $6 \mathrm{~mL}$ of MEM $+40 \mu \mathrm{M}$ spermine or MEM $+40 \mu \mathrm{M}$ spermine $+20 \mu \mathrm{M} \mathrm{Y} 27632+5 \mu \mathrm{M}$ thiazovivin for 2 days, and the inhibitory effects of spermine were examined by observation under a microscope.

\subsection{Detection of Pluripotent Stem Cell Markers}

The expression of marker proteins in TIG-1-20, HepG2, and PIS cells (Spe-1, Spe-2, and PIS-Hep2) was examined. Cells $\left(1 \times 10^{4}\right)$ were cultured on a cover glass (product code: C1100; $13 \mathrm{~mm}$ diameter; Matunami Glass Ind. Ltd., Osaka, Japan) inserted in 24-well plates containing $2 \mathrm{~mL} /$ well MEM (TIG-1-20 only) or Cellartis DEF-CSTM 500 Culture medium for 1 day. Because Spe-1 PIS cells were non-adherent, immunostaining was performed in suspended cells in a $1.5-\mathrm{mL}$ micro-cube. Immunostaining for sex-determining region Y-box 2 (SOX2), POU domain class 5 (OCT3/4), stage-specific embryonic antigen 4 (SSEA-4), TRA1-60, and TRA1-81 proteins was performed using a hES/iPS Cell Characterization Kit (product number: ASK-3006; Applied Stem Cell Inc.) according to the manufacturer's instructions.

3T3-L24 and PIS-L24 cells were cultured on cover glasses inserted into 24-well plates containing DMEM and Stem Medium for 1 day. Immunostaining for SOX2, OCT3/4, and SSEA-1 proteins was performed with an mES/iPS Cell Characterization Kit (Product number: ASK-3005; Applied Stem Cell Inc.) according to the manufacturer's instructions. Anti-rabbit IgG goat antibodies conjugated with Cy-3 and anti-mouse IgG rabbit antibodies conjugated with Alexa Fluor 555, which were diluted 1/400 with phosphate-buffered saline (PBS) containing $0.1 \%$ bovine serum albumin (BSA), were used as secondary antibodies instead of the secondary antibodies provided by the kits. After immunostaining, cells were observed with a fluorescence microscope. The expression levels of $c M Y C, S O X 2, O C T 3 / 4$, and Kruppel-like factor 4 (KLF4) in TIG-1-20, Spe-2, HepG2, and PIS-Hep2 cells were also determined by real-time polymerase chain reaction (PCR).

\subsection{Analysis of DNA Methylation}

Ratios of DNA methylation in TIG-1-20, Spe-2, HepG2, and PIS-Hep2 cells were examined. Chromosomal DNA was extracted from cells with a NucleoSpin Tissue kit (product code: U0952A; Macherey-Nalgen GmbH \& Co., Düren, Germany), and ratios of methylation in chromosomal DNA were determined with a Methylamp Global DNA Methylation Quantification Ultra Kit (product code: \#P-1014B; Epigentek Group Inc., Farmingdale, NY, USA). Percentages of DNA methylation were calculated using positive and negative controls provided by the kits. The expression of the DNA (cytosine-5)-methyltransferase 1 (DNMT1) gene in TIG-1-20, Spe-2, HepG2, and PIS-Hep2 cells was also determined by real-time PCR.

\subsection{Differentiation of Spe-2 PIS Cells into Ectoderm, Mesoderm, and Endoderm}

Differentiation of Spe-2 PIS cells into ectoderm, mesoderm, and endoderm was performed using a StemXVivo Ectoderm Kit, StemXVivo Mesoderm Kit, and StemXVivo Endoderm Kit (product code: \#SC031B, \#SC030B, and \#SC019B; R \& D Systems, Inc.), respectively. Spe-2 PIS cells (5000 cells) were cultured in 24-well plates containing $2 \mathrm{~mL} /$ well Cellartis DEF-CSTM 500 Culture medium for 1 day. The cells were further cultured with differentiation medium according to the manufacturer's instructions. The obtained cells were fixed in PBS containing 4\% formaldehyde (product code: 163-20145; Wako Pure Chemical Industries, Ltd.) for $30 \mathrm{~min}$, washed twice with $2 \mathrm{~mL}$ PBS, incubated with PBS containing $0.3 \%$ TritonX-100 and 1\% BSA for $45 \mathrm{~min}$, and washed twice with PBS. Anti-human OTX2, anti-brachyury, and anti-SOX17 goat antibodies provided by the kits were diluted in PBS containing 0.3\% TritonX-100 and $1 \%$ BSA and were used as primary antibody solutions. Cells differentiated into ectoderm, mesoderm, and endoderm were incubated with $400 \mu \mathrm{L}$ of each primary antibody solution for $16 \mathrm{~h}$ at $4^{\circ} \mathrm{C}$. Anti-goat IgG donkey antibody conjugated with Alexa Fluor 555, which was diluted 1/400 in PBS containing 0.1\% BSA, was used as secondary antibody solution. Cells were then washed with PBS four times and $400 \mu \mathrm{L}$ second- 
ary antibody solution was added; cells were further incubated for $1 \mathrm{~h}$ and washed four times with PBS. After immunostaining, cells were observed with a fluorescence microscope.

\subsection{Differentiation into Cardiomyocytes}

Differentiation into cardiomyocytes was performed with a PSdif-Cardio Cardiomyocyte Differentiation Kit (product code: PC-STR; StemRD, Burlingame, CA, USA). A, B, C, and PSGro media provided by the kit were used for the induction of mesendoderm, cardiac mesendoderm, and premyocardial cells and for the culture of myocardial cells, respectively. Spe-2 cells cultured in 24-well plates were cultured in A, B, or C media for 1, 2, or 3 days and then cultured in PSGro medium for 7 - 10 days. The obtained cells were removed with TrypLE Select and cultured in 96-well plates containing ReproCardio 2 culture medium (product code: RCD101; ReproCell Inc.). The morphological characteristics and movement of the cells cultured in ReproCardio 2 culture medium were compared with those of human cardiomyocytes (ReproCardio 2) under a microscope. The expression levels of TNN2 and NK2 homeobox 5 (NKX2 -5) genes in cells cultured in ReproCardio 2 culture medium was examined by real-time PCR.

Cells cultured with PSGro medium for 2 days were used for immunostaining. Briefly, cells in 24-well plates were fixed with PBS containing 4\% formaldehyde for $30 \mathrm{~min}$, washed twice with $2 \mathrm{~mL}$ PBS, incubated with PBS containing 0.5\% TritonX-100 and 1\% BSA for $10 \mathrm{~min}$, and washed twice with PBS. The cells were then incubated with $1 \mathrm{~mL}$ PBS containing $1 \% \mathrm{BSA}$ for $1 \mathrm{~h}$ at $30^{\circ} \mathrm{C}$ and washed twice with PBS. Anti-cTnT monoclonal antibodies and anti-sarcomere $\alpha$-actinin polyclonal antibodies, which were diluted 1:200 in PBS containing 0.1\% BSA, were used as primary antibodies. Wells were washed four times with PBS, and the cells were incubated with $400 \mu \mathrm{L}$ of each primary antibody solution for $16 \mathrm{~h}$ at $4^{\circ} \mathrm{C}$. Anti-rabbit IgG goat antibodies conjugated with Cy-3 and anti-mouse IgG rabbit antibodies conjugated with Alexa Fluor 555, which were diluted 1:400 in PBS containing 0.1\% BSA, were used as secondary antibody solutions. Wells were then washed with PBS four times, and cells were incubated with $400 \mu \mathrm{L}$ of the secondary antibody solution for $1 \mathrm{~h}$ at $30^{\circ} \mathrm{C}$, followed by four washes with PBS. Finally, the cells were observed by fluorescence microscopy.

\subsection{Differentiation into Brown Adipocytes}

Differentiation of Spe-2 PIS cells into brown adipocytes was performed with a PSdif-Cardio Brown Adipocyte Differentiation Kit (product code: PB-STR; StemRD). A, B, C, and PSGro media provided by the kit were used for the induction of mesoderm, hematopoietic precursors, brown adipocyte progenitors, and mature brown adipocytes, respectively. Spe-2 PIS cells were cultured in 24-well plates containing 1 $\mathrm{mL} /$ well Cellartis DEF-CSTM 500 Culture medium for 1 day. The cells were then cultured in A, B, or C media supplied by the kit for 1, 2, or 3 days and finally cultured in PSGro medium for 1 - 2 weeks.

The obtained cells were used for immunostaining. Briefly, cells (2000 cells) were cultured on cover glasses inserted into 24-well plates containing $2 \mathrm{~mL} /$ well PSGro medium for 1 day. The procedures for fixing, permeabilizing, blocking, and washing were the same as those described above for cells differentiated into cardiomyocytes. Anti-UCP1 rabbit polyclonal antibodies and anti-rabbit IgG goat antibodies conjugated with Cy-3, which were diluted 1:200 and 1:400 in PBS containing 0.1\% BSA, were used as primary and secondary antibodies. Immunostained cells were observed with a fluorescence microscope. The expression of the $U C P 1$ gene in the cells was examined by real-time PCR.

Accumulation of oil droplets was also examined. The obtained cells were cultured in 24-well plates containing $4 \mathrm{~mL} /$ well MEM $+0.2 \mathrm{mM}$ oleic acid for $16 \mathrm{~h}$, and oil droplets were stained with Oil Red O (product code: 154-02072; Wako Pure Chemical Industries, Ltd.), as follows. Briefly, Oil Red O saturated in isopropanol was diluted to $60 \%$ with distilled water and filtered with a $0.2-\mu \mathrm{m}$ sterile filter $(60 \%$ Oil Red O solution). The cells were fixed in PBS containing 4\% formaldehyde for 30 min, wash twice with $2 \mathrm{~mL}$ PBS, and incubated with $60 \%$ isopropanol solution for $1 \mathrm{~min}$. The cells were incubated with $60 \%$ Oil Red O solution for $20 \mathrm{~min}$ and washed three times with PBS. Oil droplets in the cells (stained red) were observed with a microscope. 


\subsection{Differentiation into Nerve Cells}

Poly-L-lysine-coated 35-mm dishes (product code: 400-040; AGC Techno Glass Co., Ltd., Shizuoka, Japan) and 24 -well plates precoated with $0.01 \%$ poly-N-ornithine solution (product code: A-004-M; Sigma-Aldrich Japan) were used for the culture and immunostaining of human PIS cells, respectively. Spe-2 PIS cells were cultured in dishes or 24-well plates containing $2 \mathrm{~mL} /$ well STEMdiff Neural Induction Medium (product code: \#05835; Veritas Corp., Tokyo, Japan) for 2 weeks. For culture of PIS-L24 cells, 24-well plates were coated with StemSure $0.1 \%(\mathrm{w} / \mathrm{v})$ gelatin solution (product code: 19010805; Wako Pure Chemical Industries, Ltd.), and cells were cultured in $2 \mathrm{~mL} /$ well NDiff 227 (product code: Y40002; Takara Bio Inc.) for 3 days, followed by culture with Complete NeuroCult NSC differentiation medium (mouse; Veritas Corp.) for 7 days. The obtained cells were examined by immunostaining using the same procedures for fixing, permeabilization, blocking, and washing as those described for cells differentiated into cardiomyocytes. Anti- $\beta$-tubulin III, anti-MBP, and anti-MAP2 rabbit polyclonal antibodies, which were diluted 1:200 in PBS containing 0.1\% BSA, were used as primary antibodies, and anti-rabbit IgG goat antibody conjugated with Cy-3, which was diluted 1:400 in PBS containing 0.1\% BSA, was used as a secondary antibody. The cells were observed with a fluorescence microscope. Additionally, the expression of $\beta$-tubulin III and MAP2 genes in cells cultured on poly-L-lysine-coated plates was examined by real-time PCR.

\subsection{Gene Expression}

Real-time PCR was used to characterize PIS cells, owing to the high specificity of the DNA primers to the target mRNA and accuracy in quantitative analysis of gene expression levels, using QuantiTect Primer Assays (Qiagen, Hilden, Germany). TIG-1-20 fibroblasts, HepG2 cancer cells, PIS cells (Spe-1, Spe-2, and PIS-Hep2), and cells differentiated into cardiomyocytes, brown adipocytes, and nerve cells were used for gene expression analyses. Total mRNA $(0.1-0.8 \mu \mathrm{g})$ was purified using an RNeasy Lipid Tissue Mini kit (Qiagen), and cDNA was synthesized using a QuantiTect Reverse Transcription kit (Qiagen). Real-time PCR was performed using a Rotor-Gene device (Qiagen). QuantiTect Primer Assays (Table 1) and a Rotor-GeneSYBR Green PCR Kit was used as DNA primers and PCR solution, respectively. According to the procedure of the kit, the reaction was carried out for $50-80$ cycles of $95^{\circ} \mathrm{C}$ for $5 \mathrm{~s}$ and $65^{\circ} \mathrm{C}$ for $30 \mathrm{~s}$. The

Table 1. List of DNA primers (QuantiTect Primer Assays) used for real-time PCR.

\begin{tabular}{cccc}
\hline Symbol & Official name & Gene ID $^{\mathrm{a}}$ & Product code $^{\mathrm{b}}$ \\
\hline ACTB & actin, beta & 60 & QT01680476 \\
SOX2 & SRY (sex determining region Y)-box 2 & 6657 & QT00237601 \\
OCT3/4 & POU class5 homeobox 1 & 5460 & QT00210840 \\
KLF4 & Kuppel-like factor 4 & 9314 & QT00061033 \\
c-Myc & v-Myc avian myelocytomatosis viral oncogene homolog & 4609 & QT00035406 \\
DNMT1 & DNA (cytosine-5-)methyltransferase & 1786 & QT00034335 \\
TNN2 & cardiac troponin T type 2 & 7139 & QT00089782 \\
NKX2-5 & NK2 homeobox 5 & 1482 & QT00010619 \\
UCP1 & uncoupling protein 1 & 7350 & QT00040915 \\
TUBIII & beta 3 tubulin & 10381 & QT00083713 \\
MAP2 & microtubule-associated protein 2 & 4133 & QT00057358 \\
\hline
\end{tabular}

${ }^{\mathrm{a}}$ Gene ID from NCBI, ${ }^{\mathrm{b}}$ Product codes of QuantiTect Primer Assays (Qiagen). 
Ct value (threshold line 0.1 ) was determined using $\beta$-actin as a standard, and the $\Delta \Delta \mathrm{Ct}$ value was calculated using the $\Delta \mathrm{Ct}$ value of TIG-1-20 cells as a standard.

\subsection{Statistical Analysis}

More than three independent experiments were performed for each test to confirm the reproducibility of the results. Results are presented as means \pm standard deviations (SDs). Statistical evaluations were conducted using two-sided t-tests, performed in Excel. Exposures of immunostaining images, taken under a fluorescence microscope (EVOS FL Auto Cell Imaging System) under the same conditions, were adjusted using similar parameter values with Photoshop CS6 software.

\section{RESULTS}

\subsection{Effects of High Spermine Concentrations on Fibroblasts}

Concentrations of polyamines are maintained at low levels in normal cells, although cancer cells produce high concentrations of polyamines $[9,10]$. Accordingly, the effects of high concentrations of polyamines on normal fibroblasts were examined. Preliminary experiments showed that the growth rate of TIG-1-20 cells gradually decreased during approximately 2 months of subculture and finally plateaued at PDL 50-55, suggesting that the TIG-1-20 cells used in this study were normal. Moreover, additional preliminary experiments were performed to determine the optimal concentration of polyamines. The results showed that cells cultured in the presence of high concentrations of putrescine, spermidine, and spermine $(10-60 \mu \mathrm{M})$ showed changes in shape, containing many small particles, and detached from the dishes; such changes were not observed at lower concentrations. Spermine showed the strongest effect, while putrescine had the weakest effect among the three polyamines. The minimum effective amount of spermine required to induce a change in cell shape was $100-200 \mathrm{nmol}$ per $1 \times 10^{5}$ cells. Accordingly, TIG-1-20 fibroblasts and spermine were selected as the optimal cell line and compound for subsequent experiments.

Next, the effects of high concentrations of spermine on morphological and physiological changes in TIG-1-20 fibroblasts were examined in further detail. Morphological changes in cells cultured in MEM containing $40 \mu \mathrm{M}$ of spermine for 1 day are shown in Figures 1(A)-((a)-(c))). Notably, small particles (around $0.2-0.5 \mu \mathrm{m}$ in diameter) contained in cells (Figure 1(A)-(b)) gradually increased by $12 \mathrm{~h}$ of culture, and many of the cells containing these particles had detached from the dish by $24 \mathrm{~h}$ of culture owing to the loss of adherent characteristics (Figure 1(A)-(c)). The AP activities of these cells were also examined, as various types of pluripotent stem cells, including ES and embryonic germ (EG) cells, show high AP activity [21]. Cells with AP activity were stained red. As shown in Figure 1(A)-(d), TIG-1-20 fibroblasts exhibited low AP activity. However, treatment with spermine increased AP activity (Figure 1(A)-(e)), and cells cultured for $24 \mathrm{~h}$ contained many small spheres showing high AP activity (Figure 1(A)-(f)). These results suggest that high concentrations of spermine induced the dedifferentiation of fibroblasts into stem cells; these cells were designated PIS cells.

\subsection{Physiological Characteristics of PIS Cells}

The physiological characteristics of PIS cells were further examined to determine whether PIS cells showed characteristics similar to pluripotent stem cells. Through a procedure described above, Spe-1 PIS cells were obtained from TIG-1-20 fibroblasts by culture with $40 \mu \mathrm{M}$ spermine. Gene expression levels in Spe-1 PIS cells were analyzed using real-time PCR. Figure 1(B) shows the ratios of $S O X 2, O C T 3 / 4$, and KLF4 mRNAs expressed in Spe-1 PIS cells to those in TIG-1-20 cells (control). High expression of SOX2 and $O C T 3 / 4$ is important in dedifferentiation into pluripotent stem cells $[22,23]$, and high expression of KLF4 is important for inhibition of apoptosis [24]. Notably, the average expression values of OCT3/4 and SOX2 in Spe-1 PIS cells were much higher than those in TIG-1-20 cells, while that of KLF4 was much 

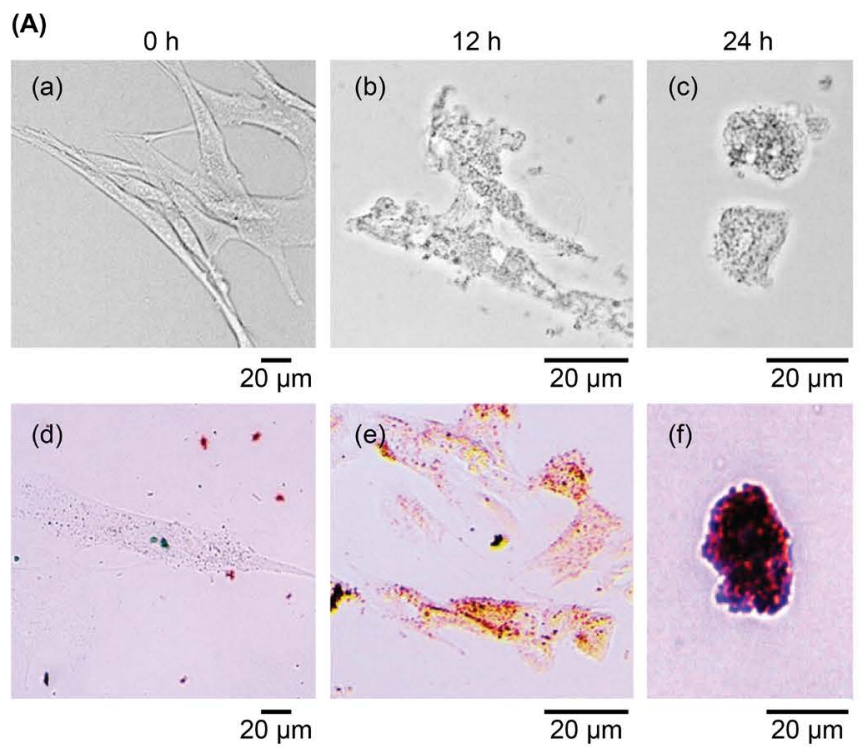

(B)

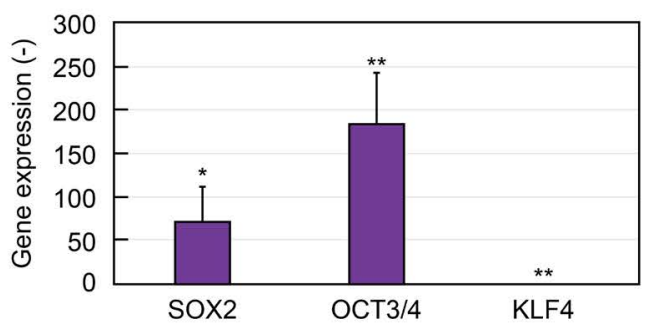

(C)

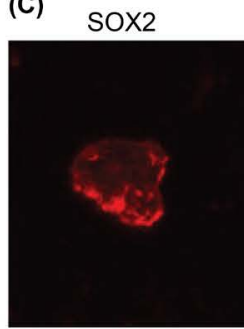

TRA1-60

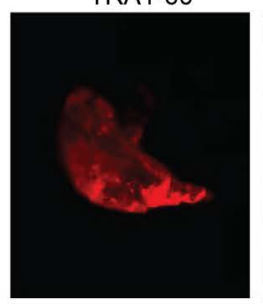

(D)

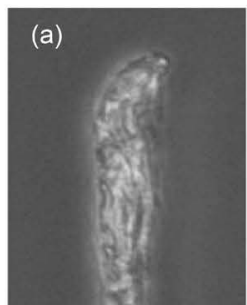

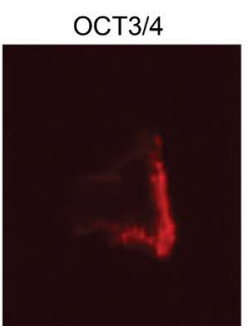

TRA1-81

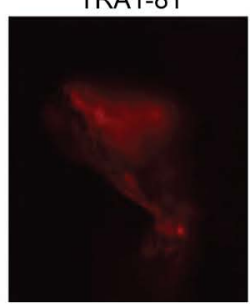

$20 \mu \mathrm{m}$
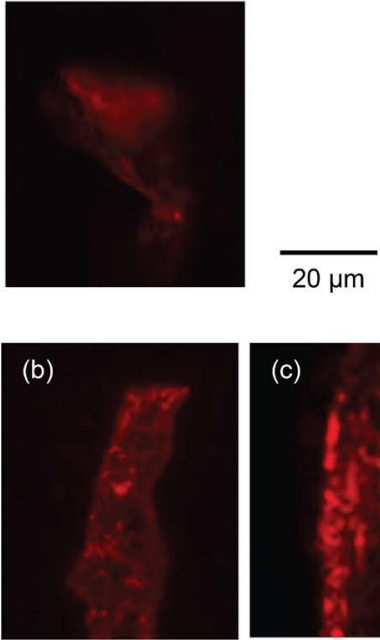
SSEA-4
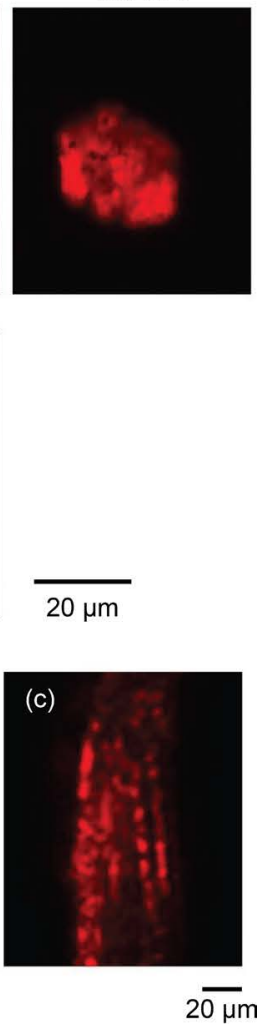

Figure 1. Dedifferentiation of TIG-1-20 fibroblasts into pluripotent stem cells induced by high concentrations of spermine. (A) Effects of high concentrations of spermine. TIG-1-20 fibroblasts were cultured in MEM containing $40 \mu \mathrm{M}$ spermine for 0,12 , or $24 \mathrm{~h}$. Photographs in the upper and lower panels show the features and AP activities of the cells, respectively. (B) Ratios of mRNAs in Spe-1 PIS cells to those in TIG-1-20 fibroblasts (control). Bars: means \pm SDs. ${ }^{* *} \mathrm{P}<0.01$ and ${ }^{*} \mathrm{P}<0.05$ versus the control $(n=3)$. (C) Immunostaining of marker proteins of pluripotent stem cells in Spe-1 PIS cells. (D) Differentiation of Spe-1 PIS cells into cardiomyocytes. (a) Features of cells. ((b) and (c)) Immunostaining with anti-cTnT antibody (b) and anti-sarcomere $\alpha$-actinin antibody (c).

lower. These results suggest that Spe-1 PIS cells are pluripotent stem cells. The results were then confirmed by analysis of SOX2, OCT3/4, SSEA-4, TRA-1-60, and TRA-1-81 protein expression. Figure 1(C) shows immunostaining results. Importantly, the expression levels of SOX2, OCT3/4, SSEA-4, TRA-1-60, and TRA-1-81 proteins in Spe-1 PIS cells were higher than those in TIG-1-20 fibroblasts.

The capacity of Spe-1 PIS cells to differentiate into cardiomyocytes was then examined (Figure 1(D)). While more than $90 \%$ of cells died during culture, many of the surviving cells showed weak vibrations (Figure 1(D)-(a)), although they did not beat with a constant rhythm and their movement was different from that of ReproCardio 2 cardiomyocytes. The expression of cTnT and sarcomere $\alpha$-actin (ACTN2) was then examined in the surviving cells (Figure 1(D)-(b) and Figure 1(D)-(c)). cTnT is specifically expressed in myocardial fibers, and the antibody used was highly specific [25]. Notably, the cells expressed cTnT (Figure 1(D)-(b)). Additionally, sarcomeres are specifically present in myofibrils of muscles and cardiomyocytes, and ACTN2 is distributed at the Z-disc portion of sarcomeres [26]. The surviving cells had sarcomere structures (Figure 1(D)-(c)). These results suggest that Spe-1 PIS cells have the capacity to differentiate into cardiomyocytes, although the obtained cardiomyocytes may include cells that are not well differentiated. Taken together, the results suggest that high concentrations of polyamines have the capacity to induce the dedifferentiation of somatic cells into pluripotent stem cells. 


\subsection{Apoptosis of PIS Cells and Effects of Rock and Apoptosis Inhibitors}

High concentrations of polyamines have been shown to induce apoptosis (3). The results of the above experiments showed that many Spe-1 PIS cells lost their adherent character during culture, suggesting that apoptosis had occurred in Spe-1 PIS cells. Thus, the effects of spermine on apoptosis in Spe-1 PIS cells were examined. Two types of Spe-1 PIS cells, which were obtained by culturing TIG-1-20 cells in medium containing $40 \mu \mathrm{M}$ spermine for $12 \mathrm{~h}$ and $36 \mathrm{~h}$, were used for this experiment.

Figure 2(A) shows the staining of TIG-1-20 and Spe-1 PIS cells (12 h) with an Apoptosis Assay kit. Spe-1 PIS cells showed evidence of cell membrane dysfunction, whereas TIG-1-20 cells did not, suggesting early apoptosis of Spe-1 PIS cells. Figure 2(B) shows the chromosomes in TIG-1-20 cells and Spe-1 PIS cells, which were stained using Hoechst 33342. Although few Spe-1 PIS cells cultured for $12 \mathrm{~h}$ exhibited fragmented chromosomes (data not shown), many chromosomes were fragmented in Spe-1 PIS cells cultured for $36 \mathrm{~h}$, suggesting that apoptosis had proceeded. Thus, these findings suggest that apoptosis occurs gradually in many Spe-1 PIS cells.

Moreover, the F-actin in Spe-1 PIS cells cultured for $36 \mathrm{~h}$ was compared with that in TIG-1-20 cells (Figure 2(C)). Cell shrinking or the depolymerization of F-actin was observed in Spe-1 PIS cells. Several studies have reported that shrinking occurs via activation of Rock and mDia proteins as Rho effectors [27, 28]. Thus, the effects of the Rock inhibitors Y-27632 and thiazovivin [16] were examined to confirm whether the Rock pathway was related to cell shrinking. Figure 2(D)-(b) shows representative images of cells cultured in medium containing Y-27632 and thiazovivin, in addition to spermine. Cell detachment and shrinking were strongly inhibited by the addition of the Rock inhibitors. These results suggest that high concentrations of spermine induce apoptosis and activation of the Rock pathway.

\subsection{Construction of New PIS Cell Line by Culture in Medium Containing Rock and Apoptosis Inhibitors}

Next, both Rock and apoptosis were inhibited, and the effects on PIS cell survival were examined. TIG-1-20 cells were cultured in Rock/apoptosis-inhibiting medium for $16 \mathrm{~h}$ before the addition of $40 \mu \mathrm{M}$ spermine and culture for another 2 days. As a result, most cells did not undergo apoptosis; however, the obtained cells did not sufficiently express markers of pluripotent stem cells and did not have the capacity to differentiate into various types of cells. These results were thought to be related to the increased number of cells in the cultures, reducing the effective amount of spermine added per cell. Therefore, the addition of higher concentrations may be necessary to produce PIS cells.

Accordingly, the optimal concentrations of spermine and treatment conditions were examined in several experiments. From this, the following improved procedure was obtained. First, $1 \times 10^{5}$ cells (PDL 30-35) were cultured in $6 \mathrm{~mL}$ Rock/apoptosis-inhibiting medium, and $20 \mu \mathrm{M}$ spermine was added. Cells were then cultured for 1 day, and $40 \mu \mathrm{M}$ spermine was added every day until the cells contained many small particles. The morphological characteristics of these cells were observed at lower (Figure 2(E)-(a)) and higher (Figure 2(E)-(b)) ratios of cell number to medium volume. Notably, PIS cells contained small particles that moved around within the cells (Figure 2(E)-(c)). These cells also attached to plate wells and grew in Cullartis DES-TM 500 Culture medium (Figure 2(E)-(d)). These newly obtained PIS cells were designated Spe-2 cells. The obtained Spe-2 PIS cells, however, showed varying expression levels of markers of pluripotent stem cells because of differences in the initial growth rate of TIG-1-20 cells; thus, five independent cell lines showing higher expression of the OCT3/4 gene were finally selected from more than 10 cell lines obtained by the culture experiments and were used for subsequent experiments.

To compare Spe-2 PIS cells with PIS cells derived from cancer cells, HepG2 hepatic cancer cells were also induced with spermine, and the obtained cell line was designated PIS-Hep2. PIS-Hep2 cells showed morphological characteristics similar to those of Spe-2 PIS cells (Figure 2(F)-(a) and Figure 2(F)-(b)). Figure 2(F)-(c) shows the cells grown in Cullartis DES-TM 500 Culture medium for 2 days. Three independent PIS-Hep2 cell lines obtained from four culture experiments were finally selected. 
(A)

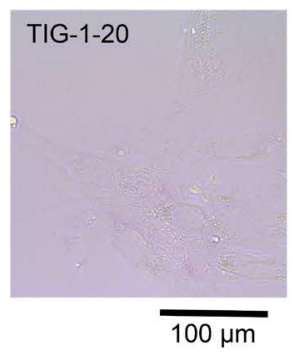

(B)

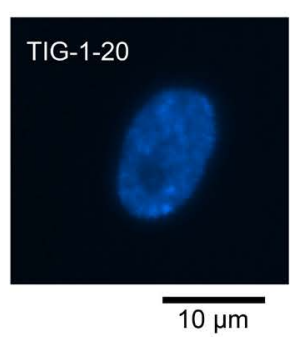

(C)

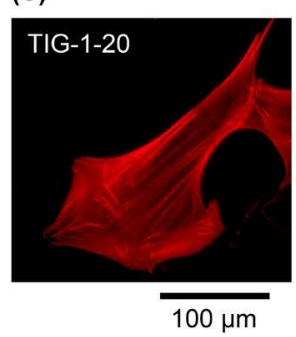

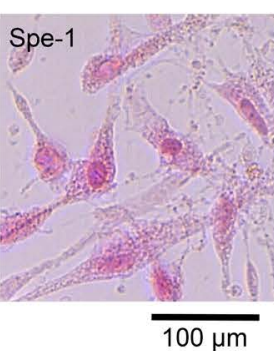
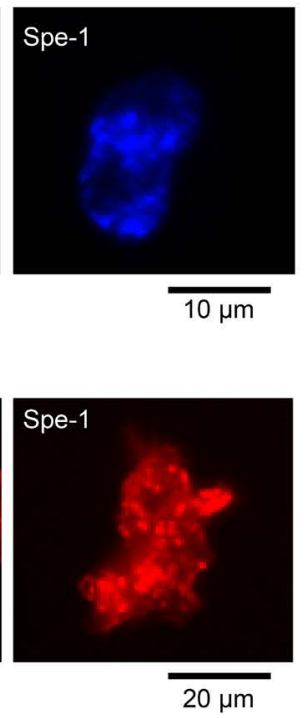

(D)

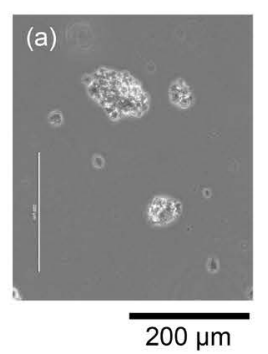

(E)

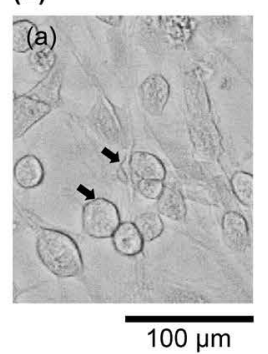

(F)

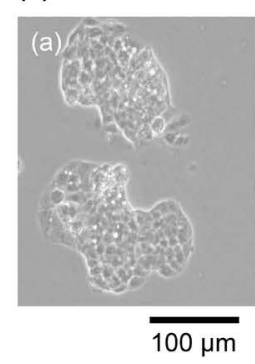

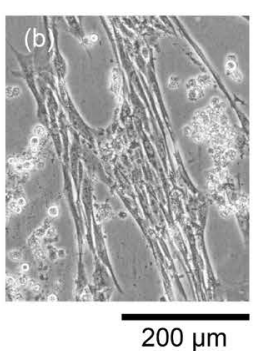
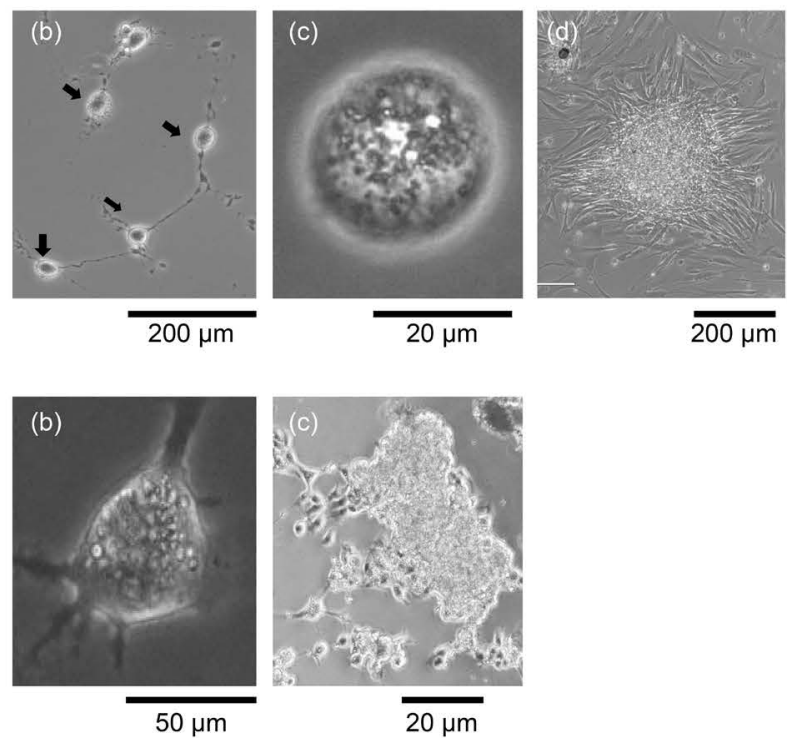

Figure 2. Effects of spermine on apoptosis of Spe-1 PIS cells and induction of new PIS cells by inhibition of apoptosis. (A) Dysfunction of the cell membrane in Spe-1 PIS cells. TIG-1-20 fibroblasts and Spe-1 cells (after culture for $12 \mathrm{~h}$ ) were stained with an apoptosis detection kit. (B) Fragmentation of chromosomes in Spe-1 PIS cells. Chromosomes of TIG-1-20 fibroblasts and Spe-1 PIS cells (after culture for $36 \mathrm{~h}$ ) were stained with Hoechst 33342. (C) Shrinking or depolymerization of F-actin. Cells were stained with Actin Red 555 reagent. (D) Effects of Rock inhibitors on shrinking or depolymerization of F-actin. (a) TIG-1-20 fibroblasts cultured in MEM $+40 \mathrm{mM}$ spermine for 3 days. (b) TIG-1-20 fibroblasts cultured in MEM $+20 \mu \mathrm{M} \mathrm{Y-27632}+5 \mu \mathrm{M}$ thiazovivin $+40 \mathrm{mM}$ spermine for 2 days. (E) New PIS cells formed by inhibition of Rock and apoptosis. ((a) and (b)) TIG-1-20 fibroblasts cultured in Rock/apoptosis-inhibiting medium containing $100 \mu \mathrm{M}$ spermine. Spe-2 PIS cells are indicated by arrows. (c) Morphological characteristics of Spe-2 PIS cells. (d) Spe-2 PIS cells cultured in Cellartis DEF-CSTM 500 culture medium for 3 days. (F) PIS-Hep2 cells derived from HepG2 cancer cells. (a) HepG2 cells. (b) Morphological characteristics of PIS-Hep2 cells. (c) PIS-Hep2 cells cultured in Cellartis DEF-CSTM 500 culture medium for 3 days.

\subsection{Pluripotent Stem Cell Characteristics of Spe-2 PIS Cells}

Next, the physiological characteristics of Spe-2 PIS cells were examined. First, the expression levels of five markers (SOX2, OCT3/4, SSEA-4, TRA-1-60, and TRA-1-81) of pluripotent stem cells were determined (Figure 3(A)). The expression levels of these five proteins were higher in HepG2 cancer cells than in TIG-1-20 cells; thus, the average exposure was decreased to equalize the band intensities. As shown in Figure 3(A), the expression levels of SOX2, OCT3/4, SSEA-4, TRA-1-60, and TRA-1-81 in Spe-2 PIS and 
(A)

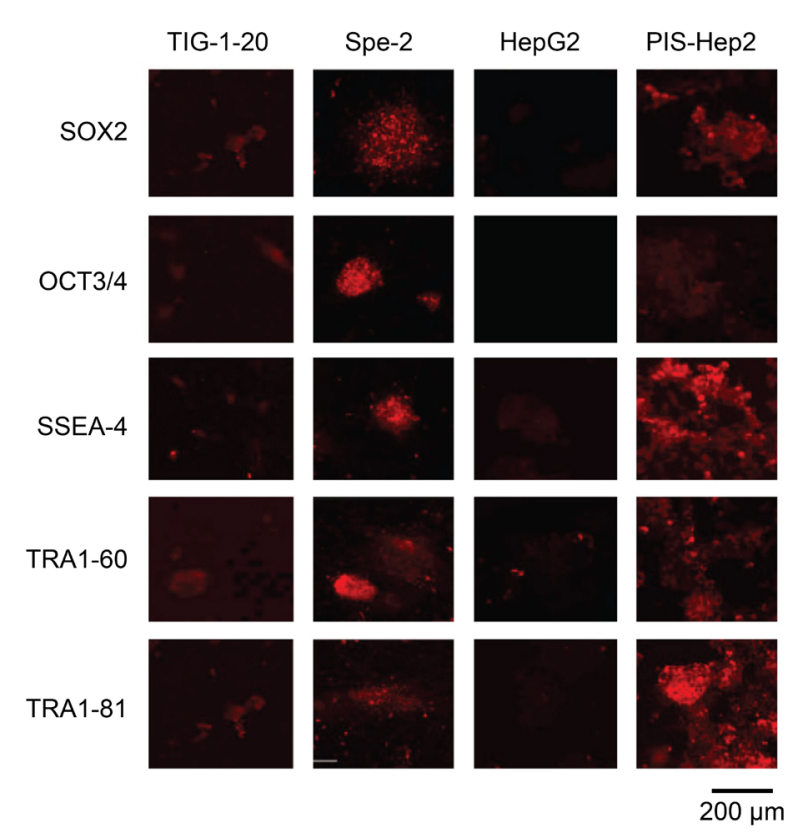

(B)

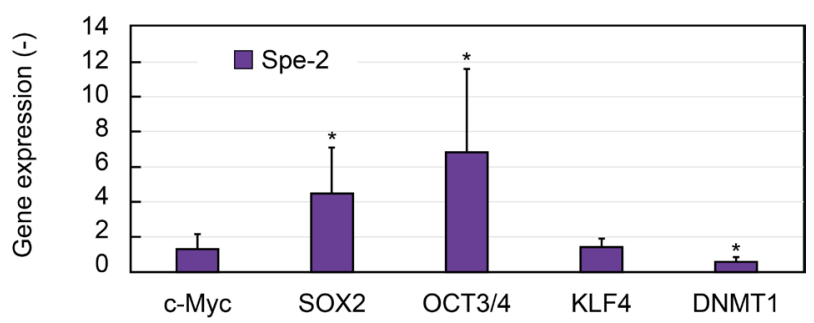

(C)

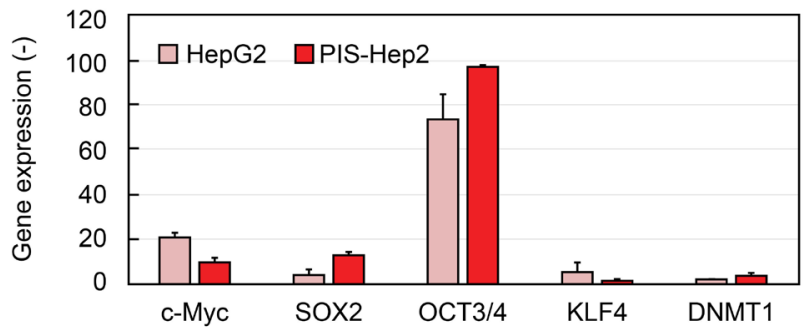

(D)

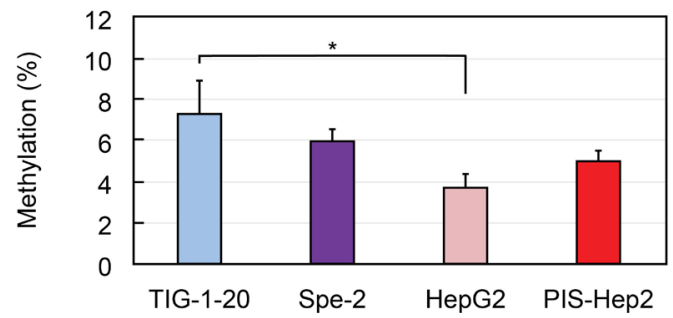

Figure 3. Characteristics of Spe-2 PIS and PIS-Hep2 cells as pluripotent stem cells. (A) Immunostaining of marker proteins of pluripotent stem cells in TIG-1-20, Spe-2, HepG2, and PIS-Hep2 cells. (B) Ratios of mRNA levels of pluripotent stem cell marker genes in Spe-2 cells to those in TIG-1-20 cells $(n=5)$. (C) Ratios of mRNA levels of pluripotent stem cell marker genes in HepG2 and PIS-Hep2 cells to those in TIG-1-20 cells $(n=3)$. (D) Percentages of DNA methylation in TIG-1-20, Spe-2 PIS, HepG2, and PIS-Hep2 cells $(n=3)$. Bars: means \pm SDs. ${ }^{*} P<0.05$ versus TIG-1-20 (control).

PIS-Hep2 cells were higher than those in TIG-1-20 fibroblasts and HepG2 cancer cells, respectively.

The gene expression levels of markers of pluripotent stem cells ( $c M Y C, S O X 2, O C T 3 / 4$, and KLF4) were also examined. Figure 3(B) and Figure 3(C) show the ratios of mRNAs expressed in Spe-2 PIS, HepG2, and PIS-Hep2 cells to those in TIG-1-20 cells. The average expression levels of SOX2 and OCT3/4 in Spe-2 PIS cells were 4.5 and 6.9 times higher than those in TIG-1-20 fibroblasts, and these increases were statistically significant ( $\mathrm{p}=0.049$ and 0.042 respectively). However, the average expression levels of cMYC and KLF4 in Spe-2 PIS cells were almost the same (1.3 and 1.4 times higher) as those in TIG-1-20 cells. The average expression levels of the five genes in HepG2 cancer cells were much higher than those in TIG-1-20 cells, and the average expression levels of SOX2 and OCT3/4 genes in PIS-Hep2 cells were higher than those in HepG2 cells.

DNA methylation ratios in pluripotent stem cells or undifferentiated cells are lower than those in normal cells because DNA methylation plays a role in maintaining the phenotypes of highly differentiated cells [29]. Thus, the DNA methylation ratios of the cell lines were examined (Figure 3(B) and Figure 3(D)). The average expression level of the DMNT1 gene, encoding DNA (cytosine 5)-methyltransferase 1, and the DNA methylation ratio in Spe-2 PIS cells were $40 \%$ and $14 \%$ lower than those in TIG-1-20 cells, respectively, suggesting that Spe-2 PIS cells were undifferentiated. In contrast, the methylated DNA ratios in HepG2 and PIS-Hep2 cells were lower than that in TIG-1-20 cells (Figure 3(D)). These results suggest 
that Spe-2 PIS cells have the potential to act as pluripotent stem cells, although this potential is lower than that of HepG2 and PIS-Hep2 cells.

\subsection{Capacity of Spe-2 PIS Cells for Differentiation into Various Cell Types}

The ability of Spe-2 PIS cells to differentiate into various types of cells was then examined to confirm their potential as pluripotent stem cells. First, Spe-2 PIS cells were differentiated into ectoderm, mesoderm, and endoderm lineages. Expression of OTX2, brachyury, and SOX17 proteins in the obtained cells was confirmed using StemXVivo kits. The results of immunostaining showed that most cells differentiated into ectoderm, mesoderm, and endoderm cells, showing strong red staining (Figures 4(A)-((a)-(c))). This

(A)

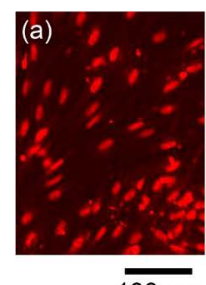

$\overline{100 \mu \mathrm{m}}$

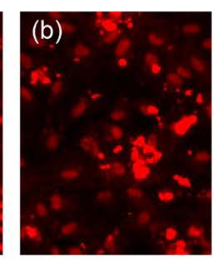

$\overline{100 \mu \mathrm{m}}$

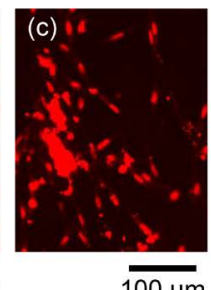

(B)
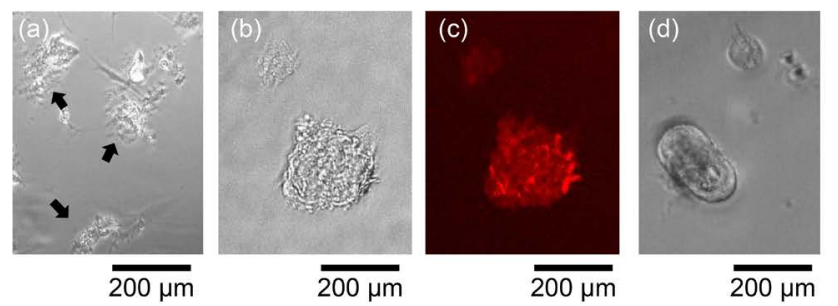

(C)
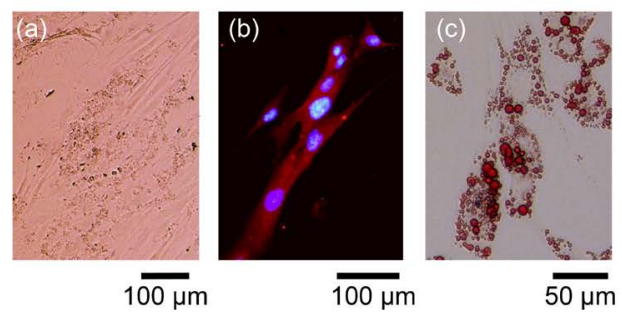

(D)

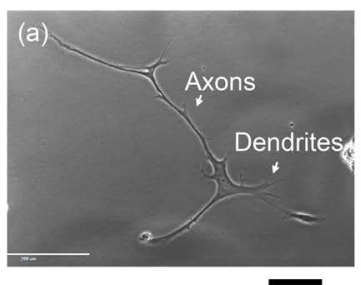

$\overline{100 \mu \mathrm{m}}$
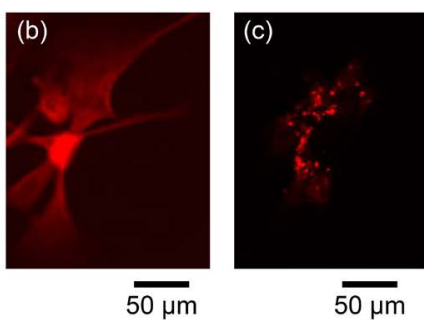

(E)

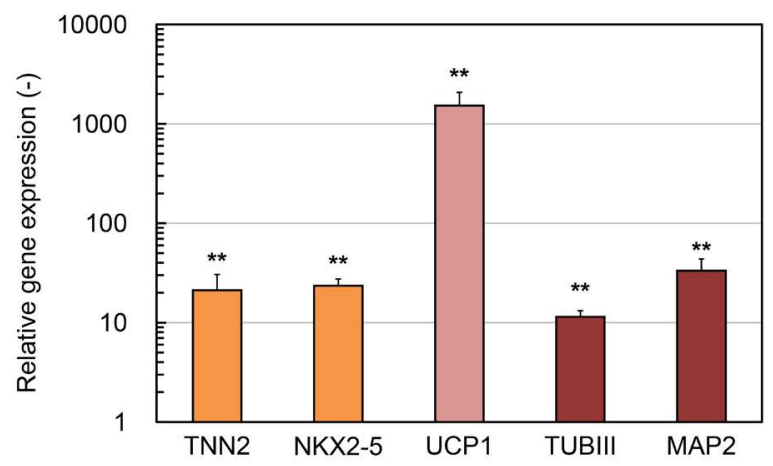

Figure 4. Capacity of Spe-2 PIS cells to differentiate into various cell types. (A) Immunostaining of cells differentiated from Spe-2 PIS cells. (a) Ectoderm. (b) Mesoderm. (c) Endoderm. (B) Characteristics of Spe-2 PIS cells differentiated into cardiomyocytes. (a) Cells cultured in C medium. Differentiated cells are indicated by arrows. (b) and (c)) Cells differentiated into cardiomyocytes, and immunostaining with anti-cTnT antibodies. (d) Cells cultured in ReproCardio 2 culture medium. (C) Characteristics of Spe-2 PIS cells differentiated into brown adipocytes. (a) Cells differentiated into brown adipocytes. (b) Immunostaining with anti-UCP1 antibodies. (c) Oil droplets stained by the Oil Red O method. Differentiated cells were cultured in MEM + $0.4 \mathrm{mM}$ oleic acid. (D) Characteristics of Spe-2 PIS cells differentiated into nerve cells. (a) Spe-2 PIS cells differentiated into progenitor nerve cells. ((b) and (c)) Immunostaining with anti-tubulin III (b) and anti-MAP2 (b) antibodies. (E) Ratio of expression levels of cardiomyocyte, brown adipocyte, and nerve cell marker genes in differentiated cells to those in TIG-1-20.fibroblasts $(n=3)$. Bars: means \pm SDs. ${ }^{*} \mathrm{P}<0.01$ versus the control (TIG-1-20). 
confirms that Spe-2 PIS cells have the capacity to differentiate into mesoderm, ectoderm, and endoderm lineages.

Next, the capacity of Spe-2 PIS cells to differentiate into cardiomyocytes via mesendoderm was examined. Figure 4(B) shows the results of differentiation into cardiomyocytes. Notably, morphological changes were observed as cells differentiated into cardiac progenitor cells (Figure 4(B)-(a)). In cells that differentiated into primary cardiomyocytes, the expression of cTnT was observed (Figure 4(B)-(b)). Further culture in differentiation medium and then growth medium for 10 days yielded cells with shapes that were similar to those of control ReproCardio 2 cardiomyocytes. Monolayers of cells (Figure 4(B)-(d)) moved rhythmically and were considered cardiomyocytes, although they did not show perfect beating motions. The expression of the TNN2 and NKX2-5 genes, important markers of cardiomyocytes, was also examined by real-time PCR. Figure 4(E) shows the ratios of mRNAs in the obtained cells to those in TIG-1-20 cells. The average expression levels of TNN2 and NKX2-5 genes in the cells were 21 and 23 times higher than those in TIG-1-20 cells, respectively. The results in Figure 4(B) and Figure 4(E) suggest that Spe-2 PIS cells have the ability to differentiate into cardiomyocytes.

Differentiation of Spe-2 PIS cells into brown adipocytes via mesoderm was then evaluated. Figure 4(C)-(a) shows cells differentiated into brown adipocytes, exhibiting brownish-stained sections. Next, the expression of UCP1, a specific marker of brown adipocytes, was examined (Figure 4(C)-(b)). Notably, the differentiated cells showed high expression of UCP1 protein. Similarly, evaluation of UCP1 gene expression (Figure 4(E)) showed that the average expression level of the $U C P 1$ gene in the cells was much higher than that in TIG-1-20 cells. Moreover, the capacity for triglyceride production in the cells was evaluated by culture in medium containing $0.2 \mathrm{mM}$ of oleic acid. Analysis with Oil Red O showed that unusually high levels of triglycerides were observed in most differentiated cells (Figure 4(C)-(c)). Similar experiments performed in TIG-1-20 fibroblasts showed that normal cells did not express UCP1 and showed reduced accumulation of oil droplets. Thus, these results suggest that Spe-2 PIS cells can differentiate into brown adipocytes [30].

Differentiation of Spe-2 PIS cells into nerve progenitor cells was then examined. As shown in Figure 4(D)-(a), after culture in neuronal differentiation medium, many cells showed axonal extensions and dendrites, exhibiting a morphology similar to that of nerve cells. Additionally, immunostaining with anti- $\beta$-tubulin III and anti-MAP2 antibodies (Figure 4(D)-(b) and Figure 4(D)-(c)) showed that differentiated cells expressed $\beta$-tubulin III and partially expressed MAP2. In contrast, TIG-1-20 fibroblasts did not express $M A P 2$ and only weakly expressed tubulin III. Moreover, expression of the TUBB3 and MAP2 genes, encoding the $\beta$-tubulin III and MAP2 proteins, was examined (Figure 4(E)). Notably, the average expression levels of TUBB3 and MAP2 were around 11 and 33 times higher than those in normal fibroblasts, suggesting that Spe-2 PIS cells can differentiate into nerve progenitor cells.

In comparison, PIS-Hep2 cells exhibited higher differentiation activity than Spe-2 PIS cells, and phenotypes were unstable. For example, after culture of PIS-Hep2 cells in Cellartis DEF-CSTM 500 Culture medium for more than 5 days, nerve-like cells spontaneously differentiated from PIS-Hep2 cells appeared (data not shown). Cells similar to adipocytes were also identified. These results suggest that high concentrations of spermine may facilitate the dedifferentiation of cancer cells and production of CSCs.

\subsection{Induction of PIS Cells from Mouse Adipocytes}

Finally, to confirm that PIS cells could be obtained in another type of cell and/or in another species, dedifferentiation of mouse adipocytes into PIS cells was examined. 3T3-L24 mouse adipocytes (20) were used. This cell line was constructed by isolating single colonies of adipocytes differentiated from 3T3-L1 pre-adipocytes; mesenchymal stem cells were not present.

3T3-L24 adipocytes were cultured in Rock/apoptosis-inhibiting medium for $16 \mathrm{~h}$, and $50 \mu \mathrm{M}$ spermine was added each day for 2 days (final concentration, $100 \mu \mathrm{M}$ ). After harvesting cells, the cells were cultured in Stem Medium for 2 - 3 days, yielding PIS-L24 cells. Figure 5(A) shows 3T3-L24 white adipocytes and PIS-L24 cells. PIS-L24 cells contained small particles, similar to Spe-2 PIS cells. The analysis of pluripotent stem cell markers (Figure 5(B)) demonstrated that the expression levels of SOX2, OCT3/4, 
(A)

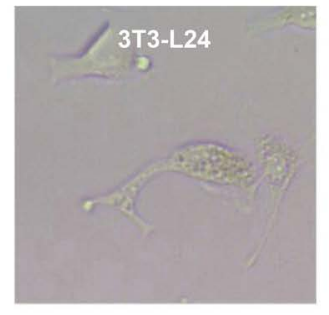

(B)
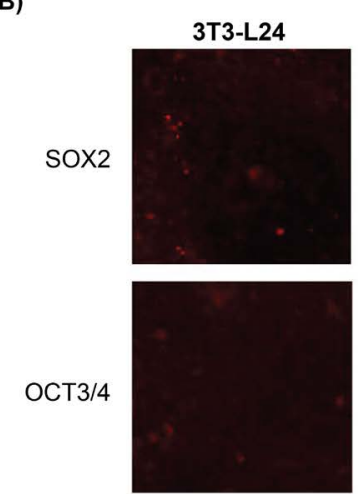

SSEA-1

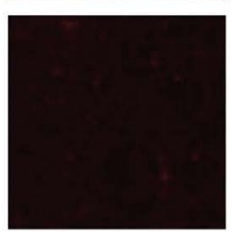

(C)
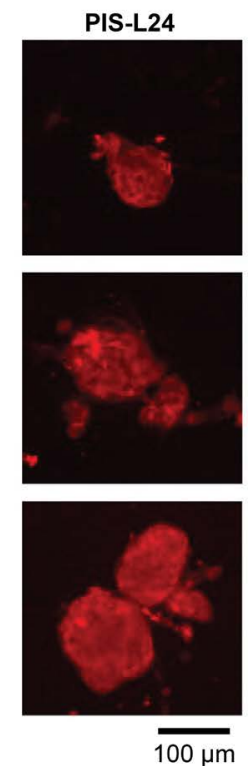

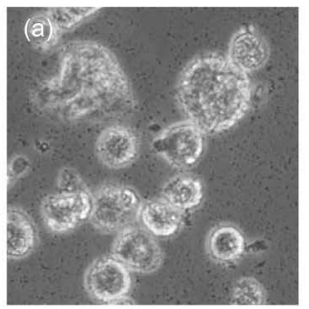

(D)

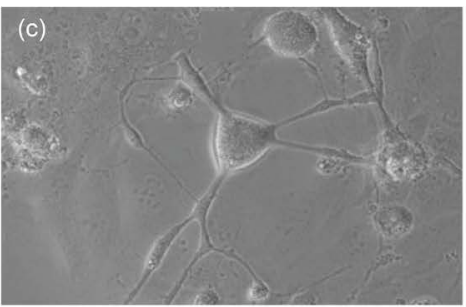

$100 \mu \mathrm{m}$
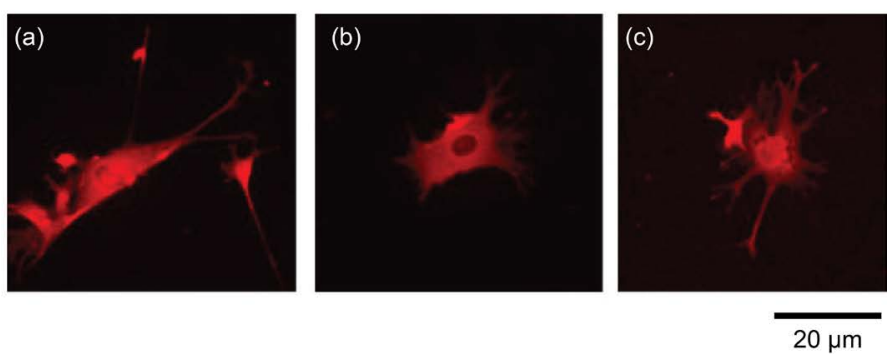

Figure 5. Induction of PIS cells from mouse white adipocytes. (A) 3T3-L24 white adipocytes and PIS-L24 cells. (B) Immunostaining of marker proteins in 3T3-L24 and PIS-L24 cells. (C) Differentiation of PIS-L24 cells into nerve cells. (a) Neurospheres. (b) Cells differentiated into nerve cells. (D) Immunostaining of the differentiated cells with anti-tubulin III (a), anti-MBP (b), and anti-MAP2 (c) antibodies.

and SSEA-1 proteins in PIS-L24 cells were much higher than those in 3T3-L24 adipocytes, suggesting that induction into PIS cells occurred in mouse adipocytes.

The differentiation of PIS-L24 cells into nerve cells was then examined to confirm the pluripotency of the cells. Following the differentiation protocol, almost all PIS-L24 cells were differentiated into neurospheres (Figure 5(C)-(a)) and then into nerve cells (Figure 5(C)-(b)). Importantly, the differentiated cells exhibited the typical shapes of nerve and/or glia cells. Furthermore, immunostaining with anti-tubulin III, anti-MAP2, and anti-MBP antibodies (Figure 5(D)) showed that these marker proteins were strongly expressed in the differentiated cells. These results suggest that mouse PIS cells differentiated from mouse adipocytes (PIS-L24 cells) have the capacity to differentiate into various cell types. Therefore, the procedure used to produce PIS cells developed in this study may be applied to cells from various species.

\section{DISCUSSION}

The purpose of this study was to elucidate the way in which cancer cells produce stem cells, with high concentrations of spermine used to investigate this mechanism. Normal somatic cells instead of cancer cells were used as a basic strain for the tests to determine the specific characteristics of polyamines, as various unknown factors are produced in cancer cells. The results provide important insights into the effects 
of polyamines on pluripotency and dedifferentiation.

The effects of high concentrations of spermine on the differentiation of normal human fibroblasts were first examined. The results showed that the expression levels of SOX2, OCT3/4, SSEA-4, TRA-1-60, and TRA-1-81 in Spe-1 PIS cells were higher than those in TIG-1-20 fibroblasts (control cells), suggesting that polyamine induced dedifferentiation of somatic cells into pluripotent stem cells. Many important roles of polyamines have been reported in previous studies [3-7]. Cheryl et al. [31] reported that high level of intercellular polyamines promoted chromatin hyperacetylation, and the results suggested that expressions of various genes might be enhanced by high level of intercellular polyamines. However, this is the first report describing the effects of high level of intercellular polyamines on dedifferentiation, highlighting the novelty of these findings.

Next, the toxicity of high concentrations of spermine was examined. The results demonstrated that at a high concentration, spermine activated the Rock pathway and induced apoptosis in normal fibroblasts. The harmful effects of spermine were inhibited upon inhibition of the Rock pathway. Cancer cells are known to be protected from apoptosis by the production of inhibitor of apoptosis proteins and/or by the mutation of tumor-suppressor genes, which inhibit proteins such as p53 and Bax. In contrast, Rock is activated in cancer cells or CSCs, facilitating the rebuilding of the cytoskeleton and enhancing invasive potential. Therefore, Rock inhibitors are expected to have applications as drugs in cancer therapy [32, 33]. The results obtained in this study and the findings of other studies $[32,33]$ suggest that normal cells constantly monitor levels of polyamines to determine whether cells are normal or abnormal, with cells containing high concentrations of polyamine removed as abnormal cells. Cancer cells, however, survive this surveillance through the production of several apoptosis inhibitors, even when cells are exposed to high concentrations of polyamines. Additionally, polyamines activate the Rock pathway. Thus, cancer cells obtain the potential for dedifferentiation, CSC production, and metastasis.

Finally, PIS cells that did not undergo apoptosis were constructed. Several studies have reported that the inactivation of Rock enhances the survival of iPS cells [34, 35]; therefore, Rock inhibitors in addition to apoptosis inhibitors were used for construction of the cells, yielding Spe-2 PIS cells. Spe-2 PIS cells expressed important pluripotent stem cell markers (SSEA-4, TRA1-60, and TRA1-80), although the expression of the $S O X 2$ and $O C T 3 / 4$ genes was only $4-8$ times higher than that in control cells. The resulting cells had the capacity to differentiate into various types of somatic cells. These results suggest that Spe- 2 PIS cells may represent a novel type of pluripotent stem cells. Additionally, in a similar model in cancer cells, PIS-Hep2 cells were found to spontaneously differentiate into other cell types, even when the medium did not contain induction factors for differentiation. This suggests that undifferentiated and pluripotent characteristics may be induced in cancer cells by high concentrations of spermine.

Importantly, the procedure used to obtain Spe-2 PIS cells in this study may be useful for regenerative medicine. iPS cells are essential for regenerative medicine [36], and several improved procedures, including transdifferentiation into cardiomyocytes without the use of iPS cells [37], have recently been reported. However, ratios of dedifferentiation remain low $(1 \%-3 \%)$ due to their dependence on the transduction efficiency of gene manipulation [38]. This makes the practical application of regenerative medicine difficult. Notably, the procedure used to obtain Spe-2 PIS cells has three advantages: 1) the procedure is fast and simple, 2) the ratio of dedifferentiation to PIS cells is much higher than that achieved using conventional procedures with genetic manipulation, and 3) large amounts of PIS cells could be produced by using adipocytes obtained from liposuction. Therefore, the procedure to obtain Spe-2 PIS cells may have remarkable potential in practical applications, particularly regenerative medicine. However, as Sep-2 PIS cells may exhibit reduced pluripotency compared to iPS cells, additional studies are necessary to enhance the capacity for dedifferentiation by determining the optimal concentration of spermine. Moreover, it is hypothesized that Spe-2 PIS cells maintain their characteristics as normal cells due to the limit of division. However, further studies to confirm this may be necessary.

\section{ACKNOWLEDGEMENTS}

This work was partially supported by Grant-in-Aids from school of Human Sciences and Laboratories 
at Kobe College.

\section{CONFLICTS OF INTEREST}

The author declares no conflicts of interest regarding the publication of this paper.

\section{REFERENCES}

1. Pegg, A.E. (2009) Mammalian Polyamine Metabolism and Function, IUBMB Life, 61, 880-894. https://doi.org/10.1002/iub.230

2. Minois, N., Carmona-Gutierrez, D. and Madeo, F. (2011) Polyamines in Aging and Disease, Aging, 3, 716-732. https://doi.org/10.18632/aging.100361

3. Miller-Fleming, L., Olin-Sandoval, V., Campbell, K. and Ralser, M. (2015) Remaining Mysteries of Molecular Biology: The Role of Polyamines in the Cell. Journal of Molecular Biology, 427, 3389-3406. https://doi.org/10.1016/j.jmb.2015.06.020

4. Igarashi, K. (2006) Physiological Functions of Polyamines and Regulation of Polyamine Content in Cells. Yakugaku Zasshi, 126, 455-471. (In Japanese) https://doi.org/10.1248/yakushi.126.455

5. Childs, A.C., Mehta, D.J. and Gerner, E.W. (2003) Polyamine-Dependent Gene Expression. Cellular and Molecular Life Sciences, 60, 1394-1406. https://doi.org/10.1007/s00018-003-2332-4

6. Oredsson, S.M. (2003) Polyamine Dependence of Normal Cell-Cycle Progression. Biochemical Society Transactions, 31, 366-337. https://doi.org/10.1042/bst0310366

7. Rhee, H.J., Kim, E.J. and Lee, J.K. (2007) Physiological Polyamines: Simple Primordial Stress Molecules. Journal of Cellular and Molecular Medicine, 11, 685-703. https://doi.org/10.1111/j.1582-4934.2007.00077.x

8. Wallace, H.M., Fraser, A.V. and Hughes, A. (2003) A Perspective of Polyamine Metabolism. The Biochemical Journal, 376, 1-14. https://doi.org/10.1042/BJ20031327

9. Linsalata, M., Caruso, M.G., Leo, S., Guerra, V., D’Attoma, B. and Di Leo, A. (2002) Prognostic Value of Tissue Polyamine Levels in Human Colorectal Carcinoma. Anticancer Research, 22, 2465-2459.

10. Löser, C., Fölsch, U.R., Paprotny, C. and Creutzfeldt, W. (1990) Polyamine Concentrations in Pancreatic Tissue, Serum, and Urine of Patients with Pancreatic Cancer. Pancreas, 5, 119-127. https://doi.org/10.1097/00006676-199003000-00001

11. Soda, K. (2011) The Mechanisms by Which Polyamines Accelerate Tumor Spread. Journal of Experimental and Clinical Cancer Research, 30, 95. https://doi.org/10.1186/1756-9966-30-95

12. Kreso, A. and Dick, J.E. (2014) Evolution of the Cancer Stem Cell Model. Cell Stem Cell, 14, 275-391. https://doi.org/10.1016/j.stem.2014.02.006

13. Nassar, D. and Blanpain, C. (2016) Cancer Stem Cells: Basic Concepts and Therapeutic Implications. Annual Review of Pathology, 11, 47-76. https://doi.org/10.1146/annurev-pathol-012615-044438

14. Bozorgi, A., Khazaei, M. and Khazaei, M.R. (2015) New Findings on Breast Cancer Stem Cells: A Review. Journal of Breast Cancer, 18, 303-312. https://doi.org/10.4048/jbc.2015.18.4.303

15. Uehata, M., Ishizaki, T., Satoh, H., Ono, T., Kawahara, T., Morishita, T., Tamakawa, H., Yamagami, K., Inui, J., Maekawa, M. and Narumiya, S. (1997) Calcium Sensitization of Smooth Muscle Mediated by a Rho-Associated Protein Kinase in Hypertension. Nature, 389, 990-994. https://doi.org/10.1038/40187

16. Lin, T., Ambasudhan, R., Yuan, X., Li, W., Hilcove, S., Abujarour, R., Lin, X., Hahm, H.S., Hao, E., Hayek, A. and Ding, S. (2009) A Chemical Platform for Improved Induction of Human iPSCs. Nature Methods, 6, 805-808. https://doi.org/10.1038/nmeth.1393

17. Yoshida, T., Tomioka, I., Nagahara, T., Holyst, T., Sawada, M., Hayes, P., Gama, V., Okuno, M., Chen, Y., Abe, 
Y., Kanouchi, T., Sasada, H., Wang, D., Yokota, T., Sato, E. and Matsuyama, S. (2004) Bax-Inhibiting Peptide Derived from Mouse and Rat Ku70, Biochemical and Biophysical Research Communications, 321, 961-966. https://doi.org/10.1016/j.bbrc.2004.07.054

18. Gudkov, A.V. and Komarova, E.A. (2005) Prospective Therapeutic Applications of p53 Inhibitors. Biochemical and Biophysical Research Communications, 331, 726-736. https://doi.org/10.1016/j.bbrc.2005.03.153

19. Ohashi, M., Aizawa, S., Ooka, H., Ohsawa, T., Kaji, K., Kondo, H., Kobayashi, T., Noumura, T., Matsuo, M., Mitsui, Y., Murota, S., Yamamoto, K., Ito, H., Shimada, H. and Utakoji, T. (1980) A New Human Diploid Cell Strain, TIG-1, for the Research on Cellular Aging. Experimental Gerontology, 15, 121-133. https://doi.org/10.1016/0531-5565(80)90083-2

20. Shiomi, N., Maeda, M. and Mimura, M. (2011) Compounds That Inhibit Triglyceride Accumulation and TNF $\alpha$ Secretion in Adipocytes. Journal of Biomedical Sciences and Engineering, 4, 684-691. https://doi.org/10.4236/jbise.2011.411085

21. Lu, H.E., Tsai, M.S., Yang, Y.C., Yuan, C.C., Wang, T.H., Lin, X.Z., Tseng, C.P. and Hwang, S.M. (2011) Selection of Alkaline Phosphatase-Positive Induced Pluripotent Stem Cells from Human Amniotic Fluid-Derived Cells by Feeder-Free System. Experimental Cell Research, 317, 1895-1903. https://doi.org/10.1016/j.yexcr.2011.05.017

22. Takahashi, K., Tanabe, K., Ohnuki, M., Narita, M., Ichisaka, T., Tomoda, K. and Yamanaka, S. (2007) Induction of Pluripotent Stem Cells from Adult Human Fibroblasts by Defined Factors. Cell, 131, 861-872. https://doi.org/10.1016/j.cell.2007.11.019

23. Takashi, M. and Muramatsu, H. (2009) Carbohydrate Markers of ES Cells. Trends in Glycoscience and Glycotechnology, 21, 197-206. https://doi.org/10.4052/tigg.21.197

24. Li, Z., Zhao, J., Li, Q., Yang, W., Song, Q., Li, W. and Liu, J. (2010) KLF4 Promotes Hydrogen-Peroxide-Induced Apoptosis of Chronic Myeloid Leukemia Cells Involving the bcl-2/bax Pathway. Cell Stress \& Chaperones, 15, 905-912. https://doi.org/10.1007/s12192-010-0199-5

25. Malouf, N.N., McMahon, D., Oakeley, A.E. and Anderson, P.A. (1992) A Cardiac Troponin T Epitope Conserved across Phyla. The Journal of Biological Chemistry, 267, 9296-9274.

26. Hwang, P.M. and Sykes, B.D. (2015) Targeting the Sarcomere to Correct Muscle Function. Nature Reviews Drug Discovery, 14, 313-328. https://doi.org/10.1038/nrd4554

27. Maekawa, M., Ishizaki, T., Boku, S., Watanabe, N., Fujita, A., Iwamatsu, A., Obinata, T., Ohashi, K., Mizuno, K. and Narumiya, S. (1999) Signaling from Rho to the Actin Cytoskeleton through Protein Kinases ROCK and LIM-Kinase. Science, 285, 895-898. https://doi.org/10.1126/science.285.5429.895

28. Ishizaki, T., Morishima, Y., Okamoto, M., Furuyashiki, T., Kato, T. and Narumiya, S. (2001) Coordination of Microtubules and the Actin Cytoskeleton by the Rho Effector mDial. Nature Cell Biology, 3, 8-14. https://doi.org/10.1038/35050598

29. Altun, G., Loring, J.F. and Laurent, L.C. (2010) DNA Methylation in Embryonic Stem Cells. Journal of Cellular Biochemistry, 109, 1-6.

30. Oelkrug, R., Polymeropoulos, E.T. and Jastroch, M. (2015) Brown Adipose Tissue: Physiological Function and Evolutionary Significance. Journal of Comparative Physiology B, 185, 587-606. https://doi.org/10.1007/s00360-015-0907-7

31. Hobbs, C.A. and Gilmour, S.K. (2000) High Levels of Intracellular Polyamines Promote Histone Acetyltransferase Activity Resulting in Chromatin Hyperacetylation. Journal of Cellular Biochemistry, 77, 345-360. https://doi.org/10.1002/(SICI)1097-4644(20000601)77:3<345::AID-JCB1>3.0.CO;2-P

32. Srinivasan, S., Ashok, V., Mohanty, S., Das, A., Das, S., Kumar, S., Sen, S. and Purwar, R. (2017) Blockade of 
Rho-Associated Protein Kinase (ROCK) Inhibits the Contractility and Invasion Potential of Cancer Stem Like Cells. Oncotarget, 8, 21418-21428. https://doi.org/10.18632/oncotarget.15248

33. Wei, L., Surma, M., Shi, S., Lambert-Cheatham, N. and Shi, J. (2016) Novel Insights into the Roles of Rho Kinase in Cancer. Archivum Immunologiae et Therapiae Experimentalis, 64, 259-278. https://doi.org/10.1007/s00005-015-0382-6

34. Watanabe, K., Ueno, M., Kamiya, D., Nishiyama, A., Matsumura, M., Wataya, T., Takahashi, J.B., Nishikawa, S., Nishikawa, S., Muguruma, K. and Sasai, Y. (2007) A Rock Inhibitor Permits Survival of Dissociated Human Embryonic Stem Cells. Nature Biotechnology, 25, 681-686. https://doi.org/10.1038/nbt1310

35. Ohgushi, M., Matsumura, M., Eiraku, M., Murakami, K., Aramaki, T., Nishiyama, A., Muguruma, K., Nakano, T., Suga, H., Ueno, M., Ishizaki, T., Suemori, H., Narumiya, S., Niwa, H. and Sasai, Y. (2010) Molecular Pathway and Cell State Responsible for Dissociation-Induced Apoptosis in Human Pluripotent Stem Cells. Cell Stem Cell, 7, 225-239. https://doi.org/10.1016/j.stem.2010.06.018

36. Peng, J. and Zeng, X. (2011) The Role of Induced Pluripotent Stem Cells in Regenerative Medicine: Neurodegenerative Diseases. Stem Cell Research \& Therapy, 2, 32. https://doi.org/10.1186/scrt73

37. Qian, L., Huang, Y., Spencer, C.I., Foley, A., Vedantham, V., Liu, L., Conway, S.J., Fu, J.D. and Srivastava, D. (2012) In Vivo Reprogramming of Murine Cardiac Fibroblasts into Induced Cardiomyocytes. Nature, 485, 593-598. https://doi.org/10.1038/nature11044

38. Kiskinis, E. and Eggan, K. (2010) Progress toward the Clinical Application of Patient-Specific Pluripotent Stem Cells. The Journal of Clinical Investigation, 120, 51-59. https://doi.org/10.1172/JCI40553 